\title{
Le mariage de la carpe et du lapin ? Une sociologie politique de la Commission européenne en chantier
}

\section{Andy SMITH et Jean Joana}

\section{(2) OpenEdition}

1 Journals

\section{Édition électronique}

URL : http://journals.openedition.org/conflits/278

DOI : $10.4000 /$ conflits. 278

ISSN : $1777-5345$

Éditeur :

CCLS - Centre d'études sur les conflits lilberté et sécurité, L'Harmattan

\section{Édition imprimée}

Date de publication : 1 juin 2000

ISBN : 2-7384-9946-5

ISSN : 1157-996X

\section{Référence électronique}

Andy SMITH et Jean Joana, « Le mariage de la carpe et du lapin ? Une sociologie politique de la Commission européenne en chantier », Cultures \& Conflits [En ligne], 38-39 | été-automne 2000, mis en ligne le 20 mars 2006, consulté le 30 mars 2021. URL : http://journals.openedition.org/conflits/278 ; DOI : https://doi.org/10.4000/conflits.278

Ce document a été généré automatiquement le 30 mars 2021.

Creative Commons License 


\title{
Le mariage de la carpe et du lapin? Une sociologie politique de la Commission européenne en chantier
}

\author{
Andy SMITH et Jean Joana
}

Depuis cinq à dix années, la capacité de la Commission européenne à participer à l'intégration européenne a été régulièrement étudiée ${ }^{1}$. Toutefois, ces travaux, centrés sur les Directions générales ou sur le rôle d'entrepreneur de politiques publiques de la Commission, laisse dans l'ombre la question de la contribution des commissaires à la légitimation de l'Europe et des institutions de l'Union. Ils tendent ainsi à privilégier des aspects organisationnels ou administratifs, faisant de l'activité de représentation des commissaires une «boite noire » dont on connaît peu de chose. Dans cette perspective, le métissage entre ces analyses en termes de politiques publiques et une démarche davantage inspirée par la sociologie politique apparaît nécessaire. C'est le parti pris d'une recherche, lancée depuis un an, qui se consacre à l'analyse des représentations et des pratiques concrètes mobilisées dans le cadre de leur action par les commissaires et par les membres de leurs cabinets ${ }^{2}$. Elle vise à éclairer les logiques de formalisation et de définition des rôles constitutifs de l'activité de commissaires et leurs portées sur la manière dont l'Union européenne est représentée. L'hypothèse de ce travail est que les commissaires participent de la production d'une forme originale d'action politique, distincte de celles qui ont été étudiées dans un cadre national. Une forme originale qui oblige à réexaminer les distinctions entre " politique » et «administratif » qui ont été forgées par la science politique pour rendre compte de la structuration des «sommets » de l'Etat. Cette combinaison entre sociologie politique et analyses des politiques européennes pourrait tout avoir du mariage de la carpe et du lapin. Surtout si l'on ne se résout pas à rabattre la compréhension des institutions européennes sur les analyses produites par ailleurs à propos des institutions politiques. Le présent article, conçu comme une présentation des étapes de la recherche et d'un ouvrage à venir, vise à explorer les conditions de possibilité de cette approche. Il s'organise autour de la 
réponse à trois questions: Que sont les commissaires européens? Que font les commissaires européens? Quelle est l'Europe que représentent les commissaires européens? Que sont les commissaires européens? Jusqu'à présent, les commissaires européens ont peu fait l'objet d'approches sociographiques. A notre connaissance, seuls E. Page et L. Wouters se sont risqués à l'exercice ${ }^{3}$. Si leurs travaux apportent des informations utiles, ils n'abordent guère la question des enjeux problématiques d'une telle approche, ni celle de la portée à reconnaître à ses résultats. Au-delà des difficultés qui accompagnent tout recours à une démarche sociographique pour rendre compte de phénomènes nationaux, sa mise en oeuvre au niveau européen pose en effet des problèmes particuliers. Dans cette perspective, il nous semble nécessaire de reformuler la question posée à ce type de matériau. Ce que l'on cherche n'est pas tant de savoir "Qui sont les commissaires européens?", mais "Que sont les commissaires européens? " ${ }^{4} l$ ne s'agit pas là d'une simple coquetterie rhétorique, mais de signifier que nous cherchons plus à éclairer le rapport que les commissaires entretiennent à leur fonction, qu'à identifier les sources de leur recrutement. A cette question, une double réponse sera en effet apportée. D'abord, nous tenterons de situer l'accès aux fonctions de commissaires dans la trajectoire des acteurs concernés. Puis, nous étendrons l'analyse aux membres des cabinets qui forment leur entourage immédiat. Les commissaires : impossible objet d'une sociographie européenne? Les travaux de nature sociographique consacrés aux commissaires européens reposent sur une tentative d'analogie, plus ou moins explicite, entre ces derniers et le personnel ministériel des différents Etats membres de l'Union'. C'est notamment le cas de l'ouvrage de 1997 qu'Edward Page a consacré à la Commission dans son ensemble, en s'intéressant tout à la fois aux commissaires eux-mêmes, aux membres de leurs cabinets et aux personnels des Directions Générales. Si les informations ponctuelles qu'il fournit sont précieuses, les conditions, pour le moins, ou le bien-fondé, au pire, d'une telle analogie, doivent être interrogées, notamment en ce qui concerne la mobilisation d'une approche sociographique. Dans un premier temps, ces difficultés sont d'ordre pratique. D'abord, il n'existe pas, à notre connaissance, de document officiel de la Commission fournissant une liste exhaustive de ses membres depuis sa création ${ }^{6}$. Ensuite, la mise en œuvre d'une sociographie passe par l'accumulation de données d'ordre biographique dont la collecte, dans un cadre européen, prend des proportions démesurées. Le chercheur qui tente l'expérience, comme E. Page, en est réduit à mobiliser les informations fournies par les annuaires et dictionnaires biographiques, de type Who's who?. Les biais que peut comporter ce type de source ont d'ores et déjà été soulignés au plan national ${ }^{7}$. On les retrouve de manière plus saillante encore avec leurs équivalents européens. L'autosélection, par les personnes citées, des informations "pertinentes " pour caractériser leur trajectoire est d'autant plus forte pour des acteurs dont l'entrée à la Commission décide de leur inscription dans ce type de dictionnaire. Des précautions sont nécessaires pour l'observateur quand il analyse des données qui ne sont portées à sa connaissance que parce les acteurs qu'elles concernent les jugent rétrospectivement dignes d'intérêt. Mais les problèmes que pose la réalisation d'une sociographie des commissaires ne se limitent pas à la seule collecte des données. Les usages des informations ainsi obtenus méritent aussi d'être interrogés. Le recours à une démarche sociographique, qui tente d'expliquer les comportements des membres d'un personnel politique à partir de leurs caractéristiques sociales a acquis un statut d'évidence en sociologie politique ${ }^{8}$. Mais c'est une évidence que l'objet que constituent les commissaires européens met à mal. Nombre des données classiques mobilisées par les 
approches sociographiques apparaissent en effet inappropriées. La portée que l'on peut reconnaître à ce type d'informations dans un cadre national est inversement proportionnelle à celle qui peut lui être attribuée dans une perspective européenne. Cela n'est pas simplement dû à la traditionnelle question de la comparabilité de catégories statistiques que l'on sait étroitement tributaires des conditions historiques, sociales et politiques qui président à leur codification. C'est surtout parce qu'elles n'ont de sens que par rapport à des logiques de formation et de recrutement des personnels politiques nationaux, dont on sait qu'elles fluctuent fortement d'un pays à l'autre9 Dans cette perspective, la forte présence parmi les commissaires de fonctionnaires, d'enseignants ou de juristes que souligne E. C. Page ${ }^{10}$, nous en dit plus sur les logiques de la réussite politique dans les différents pays constitutifs de l'Union ${ }^{11}$ que sur celles qui gouvernent l'accès à un poste de commissaire européen. La difficulté à typifier et à classer le personnel politique européen a amené les observateurs à contester l'idée d'une possible "carrière politique européenne " ${ }^{12}$. Formée à partir d'une analyse des membres du Parlement de Strasbourg, l'assertion semble prendre tout son poids dès lors que l'on s'intéresse aux commissaires. Les conditions de nomination et les modalités de candidature à un poste de commissaire sont en effet difficilement saisissables. S'il ne fait pas de doute que certains candidats ne rechignent pas à se chercher des appuis, dans bien d'autres cas, les commissaires se découvrent candidats lors de leur nomination, comme ce fut le cas pour Edith Cresson en 1995 ou Chris Patten en 1999. Outre les marchandages internationaux auxquels donnent lieu ces nominations, il semble bien que ce soit la reconnaissance d'une telle nomination comme enjeu pour les acteurs qui en bénéficient qui pose problème. L'accès à un poste de commissaire peut tout autant constituer un atout recherché que la sanction d'un échec, plus ou moins marqué, au plan national. Pour autant, le détour par la sociographie peut, sur ce point apporter des éclairages utiles, dès lors que l'on tente de reformuler les questions auxquelles on prétend répondre. Une revue bibliographique des travaux de ce type menés en France et aux Etats-Unis a mis l'accent sur les questionnements divergents qui ont animé les politistes de part et d'autre de l'Atlantique ${ }^{13}$. A une tradition française focalisée sur la représentativité sociale du personnel politique répondent en effet, aux Etats-Unis, des approches qui insistent davantage sur les procédures politiques de sélection du personnel et les accointances susceptibles d'être établies entre la trajectoire antérieure, en l'occurrence professionnelle, des élus et les positions politiques auxquelles ils accèdent. $\mathrm{Si}$, on vient de le voir, le premier type d'interrogation apparaît peu pertinent pour les commissaires, l'identification des éléments qui influent sur les conditions de leur nomination sont plus faciles à appréhender. La plupart disposent en effet d'une expérience politique, souvent avec des responsabilités de nature ministérielle ${ }^{14}$. En ce qui concerne les huit commissaires au cœur de cette étude, tous ont été ministres, et parfois même chefs de gouvernement comme Dondelinger et Cresson. La nature des dossiers traités dans le cadre de ces fonctions, leurs expériences européennes -comme élu à Strasbourg, chargé des affaires communautaires au sein d'un parti ou membre d'une autre institution bruxelloise - les responsabilités internationales qu'ils ont eues à assumer sont autant d'informations qui peuvent permettre d'éclairer les logiques politiques d'accès à la Commission, celles de l'attribution des portefeuilles et le rapport de ces acteurs à leurs nouvelles fonctions. Même si des disparités sont certainement observables selon les pays, il s'agit là d'une manière d'identifier des éléments récurrents qui pèsent sur la définition du rôle de commissaire, les rythmes en fonction 
desquels ils s'imposent et d'apporter une première réponse à la question «Qu'est-ce qu'un commissaire européen? ». Le détour par l'entourage : les cabinets Ce détour pour éclairer ce qu'est un commissaire européen peut être conçu de deux manières, qui chacune conditionne le statut accordé aux membres des cabinets dans le cadre de l'analyse. En l'occurrence, ceux-ci n'ont pas été considérés comme de simples "média", susceptibles de fournir une information sur les commissaires qui nous intéressaient, mais comme faisant partie intégrante de l'objet "commissaire». Les études portant sur la Commission consacrent fréquemment un passage à ces cabinets. Le plus souvent, il s'agit d'un tableau, très descriptif, des relations que leurs membres entretiennent avec les interlocuteurs des commissaires, institutions européennes, gouvernements nationaux ou groupes d'intérêts ${ }^{15}$. Même s'il s'agit là d'un aspect important de l'activité des commissaires, que nous nous proposons d'approfondir, un préalable passe par un éclaircissement des rapports entre ces derniers et leurs conseillers. En constituant leurs cabinets, la plupart des commissaires ont jusqu'ici privilégié des personnes de leur propre nationalité ${ }^{16}$. Composé essentiellement de six ou sept membres, ce groupe de collaborateurs se divise entre ceux qui proviennent de l'extérieur de la Commission (fonctionnaires nationaux, personnel d'entreprises publiques ou privées, cadres de partis politiques) et ceux qui sont déjà fonctionnaires européens. Si chaque membre du cabinet a des dossiers spécifiques à suivre, la première catégorie tend à s'occuper des tâches de négociation et d'intermédiation générales, tandis que les fonctionnaires européens se concentrent sur les dossiers qui exigent une compétence technique spécifique. Enfin, au cabinet s'ajoute le porte-parole du commissaire qui, formellement, est membre du Service des porte-parole (SPP) de la Commission, service rattaché directement à sa Présidence. Dans bien des cas, toutefois, le porte-parole - du propre aveu de ceux que nous avons eu l'occasion de rencontrer est appelé à atténuer son appartenance au SPP au bénéfice d'un travail personnalisé pour son commissaire, qui le rapproche du cabinet ${ }^{17}$. La marge de manœuvre dont les commissaires disposent sur le plan juridique dans le recrutement de leurs conseillers, rend ce choix d'autant plus éloquent quant aux contraintes et aux ressources d'ordre social ou politique qui interviennent dans leurs décisions. L'analyse des conditions d'entrée de ces personnels dans un cabinet, les qualités qui justifient leur recrutement, à leurs yeux ou à ceux de leur "patron", nous informent en effet sur les représentations de l'activité de commissaire qui les animent. Mettre l'accent sur ce moment du recrutement ne revient en rien à postuler que les commissaires et leur entourage ont les mains libres dans la définition de leur rôle. Les interactions avec d'autres membres du collège, avec les responsables des DG, et en particulier la DG X en matière de communication, avec la presse ou les instances représentatives des gouvernements nationaux, sont certainement d'autres dimensions à prendre en compte. Mais outre que ces dimensions ont d'ores et déjà fait l'objet d'investigation - ce qui n'exclut pas que certains aspects méritent d'en être examinés à nouveau - la constitution des équipes est révélatrice du travail d'appropriation et d'adaptation que fournissent ces acteurs, lors de leur entrée à la Commission ou au cours de leur mandat ${ }^{18}$. Dans cette perspective, la méthode choisie mêle approches quantitatives et qualitatives. La réalisation d'entretiens vient en effet compléter des informations d'ordre biographique, accumulées grâce à la réalisation d'une enquête par questionnaire. Cette double démarche présente d'abord un intérêt pratique. Elle permet tout à la fois d'obtenir des informations indisponibles autrement - les membres des cabinets n'ayant pas, sauf exception, les honneurs des dictionnaires biographiques 
- et dans certains cas de relativiser le tableau parfois trop "angélique " qu'ont fait certains de nos interlocuteurs de leurs conditions d'entrée dans la Commission. Mais l'intérêt de cette double approche va plus loin. Des travaux récents consacrés aux membres de cabinets ministériels nationaux sur une base sociographique ont en effet reconstitué les trajectoires conduisant en France ces acteurs à rejoindre l'entourage d'un ministre ${ }^{19}$. Si une telle approche est riche d'enseignements sur les conditions de recrutement et de renouvellement des élites politico-administratives - en l'occurrence dans le cadre des gouvernements socialistes des années 1980-1990 - elle ne fournit que peu d'informations explicites sur les modes d'action de ces acteurs et sur les mécanismes d'apprentissage institutionnel auxquels ils ont été confrontés une fois entrés dans un cabinet. Si une telle lacune est concevable pour des structures nationales qui ont fait par ailleurs l'objet d'études approfondies dans cette direction ${ }^{20}$, on ne pouvait s'en contenter pour des institutions européennes dont les modes de fonctionnement constituent jusqu'à présent une boite noire peu explorée. Les conditions de l'entrée dans un cabinet mettent d'abord en évidence les modalités particulières selon lesquelles les dimensions politiques et techniques de la trajectoire de ces acteurs s'agencent pour décider de leur recrutement. On sait que l'engagement partisan constitue un mode d'accès aux cabinets ministériels, certes relatif, mais important $^{21}$. En ce qui concerne les cabinets des commissaires, l'affiliation partisane, voire même les opinions des candidats aux recrutements, semblent très nettement moins déterminants. Nos interlocuteurs sont ainsi nombreux à souligner la distance qui sépare leurs opinions, voire leur engagement, de celui des commissaires qu'ils ont été appelés à servir. En atteste le témoignage d'un collaborateur de C. Scrivener, disposant par ailleurs d'appuis dans l'entourage de M. Rocard, qui déclare avoir été contacté « à la fois par la voie politique et par la voie interne " ${ }^{22}$. C'est le plus souvent leur maitrise d'un dossier particulier qui justifie à leurs yeux leur recrutement au sein d'un cabinet. Mais si les considérations partisanes sont ainsi minorées, cela n'exclut pas une fidélité à la ligne idéologique ou politique définie par le commissaire. Pour un membre du cabinet Brittan, qui déclare que le commissaire "n'était pas très intéressé par nos opinions politiques. Ça a beaucoup énervé le parti conservateur en Grande Bretagne ", son action, comme celle des autres membres du cabinet, consistait à "défendre un point de vue libéral» à Bruxelles ${ }^{23}$. Si donc la relativisation de considérations partisanes dans le recrutement n'implique pas une dépolitisation de l'action que ces acteurs sont appelés à mener, c'est le sentiment d'une recomposition particulière des débats politiques ou idéologiques au niveau européen qui rend supportable à ces acteurs ces apparentes contradictions. Le deuxième élément qui marque les conditions de leur recrutement au sein d'un cabinet, tient aux contraintes qu'imposent les règles de la collégialité au sein de la Commission. C'est la clé mobilisée par ces acteurs pour rendre compte des équilibres recherchés entre compétences de spécialistes et de généralistes au sein des cabinets. Au travers des choix de collaborateurs qui sont faits, c'est en effet le souci plus ou moins affirmé d'un commissaire de prendre position sur des dossiers extérieurs à son portefeuille qui s'expriment. Pour certains membres de cabinet, leur recrutement est justifié par la volonté de leur commissaire de se constituer une équipe lui donnant les moyens de suivre les dossiers de ses collègues. Cette quasi-obligation d'omniscience est ainsi le trait le plus marquant de leur découverte de Bruxelles, notamment pour ceux qui, issus de pays pratiquant le système des cabinets, ont déjà une expérience nationale en la matière. Si, comme nous l'avons $\mathrm{vu}$, les conditions de nomination des commissaires rendent difficiles l'identification des 
logiques d'accès à la Commission, les choix de leurs collaborateurs permettent en revanche d'apprécier le regard qu'ils portent sur leur fonction. La nature des compétences valorisées, le poids de l'expérience européenne, de l'investissement politique ou partisan, sont autant d'informations sur les représentations diverses de l'activité de commissaire qui sont produites par ces acteurs. Que font les commissaires? Si le bilan des études de ce que sont les commissaires est maigre, deux types de recherche ont généré plus de connaissances sur les pratiques des commissaires européens ${ }^{24}$. Tout d'abord, à l'occasion de recherches empiriques sur la fabrication et la mise en œuvre des politiques communautaires, un courant qui s'inspire de l'analyse des politiques publiques a souligné la nature particulièrement centrifuge de la Commission dans son ensemble ${ }^{25}$. Au-delà des concurrences interadministration qui frappent toute bureaucratie, les auteurs concernés mettent en avant les effets du caractère multiculturel du personnel de cette instance, notamment en matière d'organigrammes et de gestion des carrières. Rarement abordés de front, les commissaires sont généralement présentés comme accentuant la fragmentation de la Commission en s'engageant dans une gestion sectorielle de leurs portefeuilles et en intervenant pour faire respecter "l'équilibre géographique » de la distribution des postes de direction. Tout en épousant les questions et les techniques de recherche de l'analyse des politiques publiques, un deuxième courant se focalise plutôt sur un commissaire un peu particulier: le Président de la Commission. Plus exactement, intrigués par l'écart qui sépare la position formelle du Président (primus inter pares) des observations de son rôle réel, trois chercheurs ont réalisé des études approfondies des présidences de Jacques Delors ${ }^{26}$. Si de telles études font ressortir maintes informations sur cet exemple du leadership de la Commission, en occultant les activités des autres commissaires et du collège dans son ensemble, elles tendent néanmoins à prolonger l'image d'une Commission foncièrement fragmentée. En effet, ces recherches abordent généralement les commissaires sous l'angle d'un Etat en action, donc comme des ministres qui n'ont rien d'autre à gérer que leur portefeuille. Ainsi, la nature plus ou moins communautaire du portefeuille devient la variable qui permet d'expliquer le comportement d'un commissaire. Sans nier l'intérêt de travailler sur ce qui divise le personnel de la Commission en général et les commissaires en particulier, les premiers résultats de notre enquête permettent de souligner l'apport d'une approche qui analyse les règles et les processus qui lui donnent une certaine cohérence. Sous cet angle, à la question «que font les commissaires? » toute réponse complète doit partir de leur participation au sein du collège. Nous verrons successivement qu'en organisant et en mobilisant son entourage, un commissaire développe un rapport, plus ou moins heureux, avec «sa » ou « ses » Direction(s) générale(s). En s'articulant l'une par rapport à l'autre, ces deux dimensions informent fortement la capacité d'action d'un commissaire face à ses interlocuteurs externes, notamment ceux du Conseil et du Parlement européen. Fidéliser un entourage pour se faire respecter au collège Nous avons vu plus haut qu'en choisissant son cabinet chaque commissaire tend à établir un équilibre particulier entre les compétences de ceux qui vont devenir ses plus proches collaborateurs. Une fois en place, il lui reste encore à délimiter un rôle pour son cabinet en définissant un mode de fonctionnement interne et externe, d'une part, et des manières d'entretenir la fidélité, voire la " foi ", d'autre part. Nos entretiens suggèrent que les orientations que choisit un commissaire dans ce cadre sont souvent facteur de réussite ou d'échec au niveau de son insertion au sein du collège. Sur le plan de l'emploi du temps d'un cabinet, en effet, les règles explicites et implicites de la collégialité ont 
déjà une grande importance. Comme toute décision de la Commission doit être prise lors de la réunion hebdomadaire du collège qui se déroule le mercredi, sa préparation est canalisée à travers une pléthore de réunions inter-cabinets dans lesquelles figurent soit les chefs de cabinet, soit les spécialistes d'un dossier de chaque cabinet («Special chefs»). Il s'ensuit qu'au sein de chaque cabinet certains membres travaillent plus spécifiquement sur le ou les portefeuilles de leur commissaire, et donc présentent ces dossiers dans les réunions inter-cabinets, tandis que d'autres centrent davantage leur activité sur les enjeux « hors-portefeuille » et ont par conséquent plus un rôle de veille critique sur les dossiers présentés par d'autres cabinets. Du point de vue de l'analyse des commissaires, la gestion du rapport portefeuille/hors-portefeuille nous semble offrir une clé d'interprétation féconde et ceci pour au moins trois raisons. Tout d'abord, étudier la priorité relative accordée à ces deux types de travaux permet de mieux comprendre si la représentation principale du collège tenue par chaque commissaire est plutôt celle d'une collection de spécialistes de l'action publique ou d'un groupe de généralistes à vocation politique. En concevant leur cabinet essentiellement comme une interface avec leur Direction générale (voir infra), certains commissaires se rangent très clairement dans le premier cas de figure. C'était le cas, par exemple, de Ray MacSharry, commissaire responsable de la politique agricole entre 1989 et 1992. Certes, les membres de son cabinet assistaient aux réunions inter-cabinets, mais beaucoup de ce travail a été délégué à la personne la moins expérimentée de l'équipe et au chef de cabinet, les autres réservant leur temps, leur énergie et leur capital de négociation pour les (multiples) dossiers agricoles qu'ils avaient à leur charge. D'autres commissaires, tels que Sir Leon Brittan ou Yves de Silguy, avaient une approche presque inversée des dossiers hors-portefeuille. Responsable de la politique de la concurrence (1989-92), et ensuite du commerce et des affaires extérieures (1993-99), Brittan a systématiquement structuré ses cabinets de manière à ce que les membres de son personnel travaillent autant sur leurs " propres » dossiers que sur ceux des autres commissaires. $\mathrm{Si}$, en poussant leurs collègues des autres cabinets à ajuster et/ou à améliorer leurs "copies", les membres des cabinets de Brittan n'ont pas toujours réussi à faire passer leur point de vue, ils ont néanmoins souvent gagné l'écoute, voire le respect, de leurs confrères. Comme nous l'a dit, sans se vanter, un collaborateur de Brittan de la période 1993-94, "dans les réunions inter-cabinets, les autres nous écoutaient, même s'ils n'étaient pas d'accord. Par exemple, pour eux, notre temps de parole n'était pas le moment d'aller au toilette ! Ce qui était peut-être moins le cas pour la parole de certains autres cabinets... " ${ }^{27}$. Gagner le respect des autres cabinets et de leurs commissaires a également été un des objectifs de Yves de Silguy, commissaire responsable de la politique monétaire entre 1995 et 1999. En structurant le travail de son cabinet, ce dernier a érigé en principe d'action l'idée que " pour être crédible au Conseil il faut également être crédible au Parlement européen et au collège ". ${ }^{28}$. Si mieux comprendre le rapport spécialiste-généraliste constitue un premier éclairage offert par l'étude des distributions des tâches au sein du cabinet, un deuxième concerne la manière dont chaque commissaire gère le rapport intérêt national-intérêt européen. Plus exactement, si le Traité de Rome précise qu'un commissaire doit œuvrer pour le bien commun communautaire, et donc refuser d'accepter les instructions du gouvernement de son pays d'origine, en pratique, chacun fait l'objet de pressions quotidiennes les incitant à prendre position sur des dossiers ayant un lien plus ou moins proche avec leur portefeuille. Or, en étant le lieu de réception principal de ces pressions, du point de vue de la recherche, le cabinet d'un commissaire est 
naturellement un lieu d'observation clé pour mieux comprendre les processus et les décisions qui marquent le rapport national-communautaire. Toutefois, contrairement à ce qui est souvent écrit sur ce sujet ${ }^{29}$, il est finalement assez rare qu'un commissaire défende systématiquement un intérêt "national » ou l'intérêt d'un acteur économique national. Dans un contexte de collégialité où les alliances entre commissaires sont rarement durables, un tel comportement est difficilement tenable à moyen terme. Il n'en reste pas moins que la manière dont s'organisent les cabinets offre quelques indices sur le type de travail en profondeur qui peut être fait pour avancer ou protéger les intérêts nationaux. L'exemple le plus clair ici concerne la manière dont les cabinets des commissaires français abordent les dossiers agricoles. Depuis la création de la Commission en 1958, un commissaire français n'a jamais tenu le poste de responsable de l'agriculture. Il est néanmoins plus que fréquent que les commissaires de ce pays nomment dans leur cabinet un spécialiste pour suivre ce dossier de près. Dans le cas de Christiane Scrivener, par exemple (responsable des politiques fiscales de 1989 à 1992), son chef de cabinet a recruté un spécialiste du commerce agricole international directement au Ministère de l'Agriculture à Paris. Pour sa part, Jacques Delors a toujours eu un spécialiste agricole au sein de ses cabinets, tandis qu'Yves de Silguy a fait suivre cet aspect du travail de la Commission par son chef de cabinet adjoint, un fonctionnaire européen expérimenté qui avait appris les rouages de la PAC (politique agricole commune) lorsqu'il était en poste à la Direction générale du budget. Si le suivi des dossiers hors-portefeuille est justifié par nos interviewés sous l'angle d'un besoin « normal » du collège de connaître les «sensibilités nationales » avant de prendre une décision ou de proposer une législation au Conseil, en pratique elle n'est ni dépolitisée sur le plan de son déroulement, ni anodine sur le plan de ses effets. En effet, au-delà de leur expertise technique, une partie importante des membres de cabinets sont aussi nommés pour leur connaissance de, et leur degré d'insertion dans, les réseaux nationaux de politique publique. Enfin, analyser la composition et le comportement des cabinets permet de mieux comprendre un troisième rapport, celui qui lie chaque commissaire aux co-membres du collège en général et au Président de la Commission en particulier. Plutôt que d'attribuer la qualité de ce rapport aux ressources et aux contraintes de ce "leader", il nous semble plus pertinent d'interroger les représentations de la collégialité développées par chaque présidence. Dans une telle perspective, le vote ou l'absence de vote au sein du collège offre un éclairage à la fois sur le rapport inter-commissaires et sur le rôle que peut jouer un Président. Contrairement à la situation au sein de bien des Conseils de ministres nationaux, la position d'un commissaire est frappée par une tendance paradoxale. D'un côté, il est évident que certains commissaires bénéficient de plus d'autorité que d'autres du fait de leur carrière précédente, de leur ancienneté à la Commission et de la nature de leur portefeuille. De l'autre, lorsque le collège vote, tous ses membres redeviennent des égaux. Par conséquent, on pourrait s'attendre à ce que le fait d'être membre du collège implique de garder à l'esprit le pouvoir latent de chacun de ses collègues, de tisser des alliances lors de la préparation de chaque décision et donc d'être prêt à marchander un vote ou une abstention à court terme en faveur d'un soutien futur. Ces traits ont été en effet celui de la deuxième Commission Delors (1989-92), une Commission marquée par un collège qui est souvent passé au vote et où il n'y a pas eu de pactes durables entre les commissaires sur un plan idéologique (droite-gauche), ou sur un plan sociologique (nord-sud). Toutefois, la pratique du collège présidé par Jacques Santer (1995-99) souligne que loin d'être institutionnalisé, le passage au vote est plutôt un choix qui 
découle des dispositions du Président et de son entourage. Au sein de ce collège, le passage au vote s'est très vite estompé en faveur de la fabrication de décisions consensuelles et/ou de non-décisions. Selon beaucoup de nos interlocuteurs, cette pratique a renforcé les tendances centrifuges déjà bien ancrées au sein de la Commission en facilitant l'émergence de "barons" intouchables au sein du collège. Selon un membre de cabinet de Brittan, par exemple, «le collège est devenu un club sympathique (a cosy club) où on ne voulait pas pousser les débats jusqu'à voter. En effet, une idée a émergé selon laquelle le fait de voter était 'anti-collégial'. Anticollégial mon oeil ! C'est très dangereux de laisser développer une ambiance où on ne peut pas voter. Il faut être capable de passer au vote et ensuite digérer ses défaites de manière responsable ${ }^{30}$. Le deuxième aspect du rapport entre un commissaire et son cabinet qui a retenu notre attention concerne les efforts faits par le premier pour fidéliser son entourage. Tout comme au niveau national ${ }^{31}$, au delà des gratifications symboliques associées au fait d'être membre de cabinet, l'entretien d'une telle relation passe en partie par la capacité d'un commissaire à intervenir pour assurer qu'un passage par de tels postes soit un "tremplin" qui fait avancer la suite de leurs carrières respectives. Au sein de la Commission, de telles trajectoires font effectivement partie des pratiques institutionnalisées. Dans le cas des fonctionnaires européens dans les cabinets, ceux-ci tendent à retourner dans leurs DG d'origine comme chefs d'unité, voire même comme directeurs. Dans le cas des personnes nommées aux cabinets de l'extérieur, un tel sort semble à première vue moins évident. Il n'en reste pas moins qu'en passant des "concours internes", ceux-ci peuvent souvent intégrer la Commission relativement facilement. De même, les anciens membres de cabinet préférant retourner dans leur pays d'origine tendent le plus souvent à trouver des postes de responsabilité soit au sein de la fonction publique, soit dans une entreprise ${ }^{32}$. Mais si ce qui se passera après le mandat du commissaire a sans soute une certaine importance pour fidéliser un entourage, il nous semble important de ne pas l'exagérer. Lorsqu'on est dans le feu de l'action, il importe plus qu'un commissaire échange avec son cabinet, d'une part, et développe une ligne à suivre, d'autre part. En ce qui concerne les discussions entre un commissaire et les membres de son cabinet, il semblerait qu'il y a des différences d'approche considérables. Pour certains commissaires, tels que Brittan et de Silguy, chaque journée de travail à Bruxelles a commencé par une réunion avec leur cabinet et leur porte-parole. Selon nos interviewés, ces rencontres quotidiennes étaient non seulement une occasion de présenter l'état de leurs propres dossiers aux uns et aux autres, mais aussi d'échanger des informations sur les dispositions des autres commissaires et leurs cabinets. Ainsi, par exemple, lorsque Brittan était responsable de la politique de la concurrence, son cabinet était bien placé pour calculer le nombre de votes qu'il obtiendrait au collège lors des débats souvent houleux concernant l'interdiction de fusions ou d'aides d'Etat. Par ailleurs, tant chez Brittan que chez de Silguy, on note le peu d'importance attachée à la hiérarchie interne du cabinet. Certes, dans chaque cas, les chefs de cabinets avaient un rôle clé, mais ils n'ont pas été un écran entre le commissaire et le reste de son équipe. Dans d'autres cabinets étudiés, les hiérarchies sont plus marquées et la distance entre le commissaire et certains membres de son cabinet sont plus grandes. L'exemple le plus évident de ce cas de figure est celui d'Edith Cresson, responsable des politiques de recherche et d'éducation entre 1995 et 1999. En règle générale, ce commissaire n'a passé que deux ou trois jours à Bruxelles par semaine. Les réunions de cabinets ont donc été plus rares. De plus, les membres de son cabinet ont changé plusieurs fois 
pendant son mandat (seul un fonctionnaire européen y est resté jusqu'à son terme), degré de changement reconduit au niveau de son porte-parole (trois en quatre ans et demi). Outre le degré et la qualité du contact entre un commissaire et son cabinet, afin de comprendre comment s'est fait (ou ne s'est pas fait) la fidélisation, il faut également prendre en compte la substance des dossiers à leur charge. Souvent résumée par nos interviewés comme l'établissement et puis la défense d'une "ligne politique ", la cohérence des prises de position d'un commissaire semble être un élément clé pour comprendre à la fois le degré d'engagement des membres de cabinet et leur dévouement plus ou moins grand à leur commissaire respectif. Lors de son mandat sur la concurrence, les collaborateurs de Leon Brittan mettent explicitement en avant la nature « idéologique » de leur démarche en soulignant comment leur travail collectif a contribué à normaliser («to transform into conventional wisdom») une nouvelle approche du rôle de l'Etat dans l'économie en Europe. Outre le fait de faciliter l'avancement de leurs propres dossiers, la clarté des priorités et de la méthode de leur commissaire, disent-ils, leur ont permis d'être très présents dans les réunions intercabinets qui préparaient une multitude d'autres décisions du collège. A sa façon, Edith Cresson a également développé une ligne politique en soulignant, contrairement à Brittan, le rôle de catalyseur que devraient jouer les pouvoirs publics pour provoquer le changement économique. Résumée dans son ouvrage Innover ou subir, cette conceptualisation du rôle du politique a également informé ses prises de position au collège contre la construction d'un marché transatlantique moins réglementée ${ }^{33}$. Toutefois, force est de constater qu'entre les idées qu'elle a pu développer et défendre elle-même et celles qui donnaient une ligne de conduite à son cabinet, il y a souvent eu un hiatus qui ne favorisait pas la fidélisation de son entourage. Au total, cette introduction au monde des cabinets à la Commission européenne permet de souligner l'importance pour un commissaire de produire, de structurer et de galvaniser un entourage de "fidèles" ${ }^{34}$. Tout comme au niveau national, la réputation, la perspicacité et le dynamisme d'un seul homme ou femme ne fabriquent pas un acteur puissant ou efficace. Dans des situations d'interdépendance institutionnelle forte, mener une équipe capable de participer pleinement à la médiation constitue souvent un atout considérable. Soulignons, toutefois, que si la composition et les capacités des équipes des commissaires comportent quelques similarités avec les cabinets en France (expertise technique, jugement politique, réseaux...), la nature collégiale de la prise de décision finale induit toute une série de différences, auxquelles s'ajoute un rapport particulier entre un commissaire et l'administration de la Commission. "Coiffer" une direction générale Si les commissaires et leurs cabinets sont au centre de notre enquête, il est évidemment indispensable d'étudier la manière dont ils travaillent avec la vingtaine de Directions générales (DG) qui composent l'administration de la Commission. De manière formelle, chaque commissaire est responsable au sein du collège d'un ou plusieurs "portefeuilles ", tandis que chaque DG est dirigée par son directeur général. Dans la pratique, les commissaires sont sommés de travailler de manière étroite avec la ou les DG qui correspondent à leurs portefeuilles, tendance que d'ailleurs l'actuel président de la Commission, Romano Prodi, a tenté de renforcer ${ }^{35}$. Il n'en reste pas moins que le rapport commissaire-DG est frappé d'ambiguïtés et d'incertitudes qui découlent du rôle peu stabilisé du commissaire, ce qui tend encore à le différencier d'un ministre national. En effet, un des symptômes de ce rapport est que dans le jargon communautaire, un commissaire ne commande pas ou ne gère pas une DG, il la «coiffe ». Cela étant, notre recherche montre clairement que, d'une part, 
certains commissaires établissent un rapport plus direct et plus directif avec l'administration de la Commission et, d'autre part, que la nature de ce rapport a des conséquences sur la préparation et la présentation des décisions du collège, voire de l'Union européenne dans son ensemble. Nous ne pouvons entrer ici dans un débat sur l'influence relative d'un commissaire sur une décision communautaire. Abordons plutôt une question préliminaire qui concerne les différents types de relation établis entre un commissaire et l'administration de la Commission. Comme nous l'avons indiqué plus haut, la réponse orthodoxe à cette question consiste à lier le comportement d'un commissaire à la nature plus ou moins communautaire de son portefeuille. Sous cet angle, il est sans doute vrai que le degré d'homogénéité des portefeuilles tenus par un commissaire a une influence sur son comportement. Pour ne citer que l'exemple du collège de 1995-99, des commissaires comme Edith Cresson ou Manuel Oreja Aguirre ont eu des responsabilités éclatées qui les ont emmenés à travailler avec plusieurs Directions générales ${ }^{36}$. D'autres comme Leon Brittan, Yves de Silguy et Franz Fishler se sont occupés principalement d'un seul portefeuille et ont donc été amenés à établir un rapport privilégié avec une seule $\mathrm{DG}^{37}$. Ensuite, il est indéniable que certains champs d'activité de la Commission (concurrence, commerce international, agriculture) accordent a priori une capacité d'intervention plus importante au commissaire qui en a la responsabilité. Toutefois, de tels constats généraux ne nous disent rien sur la manière dont un commissaire et/ou une Direction générale active le droit d'intervention formel de la Commission dans tel ou tel domaine. Afin d'étudier le travail fait sur ce plan par certains commissaires, il convient de considérer qu'au-delà des défis administratifs que pose la gestion d'un ou de plusieurs portefeuilles, se profile un défi politique qui consiste à donner un fil conducteur à leurs activités et ainsi à s'établir une image au sein et en dehors de la Commission. En tant qu'instance d'interface entre une DG et un commissaire, nous avons vu plus haut que la méthode et les ressources d'un cabinet est une première variable explicative des différences de rapport commissaire-DG que connait chaque Commission. L'analyse de deux autres rapports permet d'approfondir cette question. Le premier rapport est celui qui se développe entre un commissaire et le personnel de la ou des administrations qui sont ses interlocuteurs privilégiés au sein de la Commission. Un premier élément concerne la manière dont la hiérarchie de la DG est associée aux réunions entre le commissaire et son cabinet. Entre 1989 et 1992, par exemple, Leon Brittan a tenu à ce qu'un représentant de la DG IV assiste à ces réunions une fois par semaine. De manière un peu paradoxale, ce commissaire a aussi insisté pour que les fonctionnaires "de base" de la IV assistent à des réunions consacrées à des dossiers précis, tels que les interdictions de fusions, afin de dialoguer directement avec l'agent de la Commission le plus informé. Comme nous a dit un membre de son cabinet, « cette pratique a beaucoup choqué la hiérarchie de la DG IV, mais il a également beaucoup motivé les gens dans ses services" ${ }^{38}{ }^{39}$. Dès son entrée en fonction en janvier 1995, selon ses dires, Yves de Silguy a également cherché explicitement à établir un rapport de complémentarité avec les agents de la DG II. «A partir d'une première réunion avec sa direction où je leur ai demandé tout simplement quels étaient les problèmes qui pourraient empêcher la marche vers l'UEM, et où on m'a répondu qu'on avait publié les papiers dessus - auquel j'ai répliqué qu'on n'était pas là pour gagner le prix Nobel de l'économie - on s'est très bien entendu. Je leur ai demandé de décomposer ces problèmes et de me dire comment faire. Après c'était du "project management" définir les objectifs et aller vers leur réalisation ${ }^{40}$. En travaillant avec le Directeur 
général de la DG VI, ainsi qu'avec des groupes de travail qui respectaient peu la hiérarchie interne de cette administration, Ray MacSharry offre un troisième exemple des manières développées par les commissaires pour travailler avec les fonctionnaires européens. Plutôt que de continuer à énumérer ces démarches et établir une typologie, nos recherches futures cibleront la genèse et l'évolution de la manière dont un commissaire gère le rapport entre politique et expertise au cours de son mandat. Enfin, une troisième variable qui permet d'interroger et de comparer le rapport commissaireDG se résume à la manière dont chaque membre du collège s'appuie sur les ressources et les compétences de l'unité d'information de la Direction générale en question. Etablies par incréments depuis la fin des années 1970, chacune de ces unités a un statut et une taille très différente d'une DG à une autre. De plus, historiquement elles se sont développées en essayant de trouver une place entre d'un côté le Service des porteparole des commissaires et, de l'autre, la Direction générale (la DG X) spécialisée dans l'information générale sur les activités de l'Union européenne. Nos recherches montrent que certains commissaires ont cherché à mobiliser l'unité d'information leur correspondant (comme ce fut le cas d'Yves de Silguy et de celle de la DG II pour la campagne sur l'euro), tandis que d'autres ont préféré rester dans une posture critique et distancée par rapport à de telles unités en canalisant l'information via leur porteparole (comme Leon Brittan et l'Organisation mondiale du commerce). Au total, l'étude du comportement des commissaires peut servir à prolonger et à discuter l'analyse de la nature centrifuge de la Commission. Cette tendance vers l'éclatement des services et des pratiques a jusqu'ici été étudiée à travers les caractéristiques de la vie quotidienne dans les services (socialisation hétéroclite des fonctionnaires, gestion des carrières dans un contexte de postes à drapeau, hiérarchies souples...). Analyser le rapport entre un Commissaire et "sa » ou "ses " DG permet de compléter de telles analyses en soulignant que la compétition inter-DG peut s'expliquer par la non-stabilisation du rôle de commissaire dans la fabrication des prises de position de la Commission. En même temps, l'étude des commissaires en action au sein d'un collège pousse également à nuancer l'hypothèse, à notre sens trop générale, d'une Commission intrinsèquement fragmentée. Les commissaires et la représentation Un des apprentissages les plus difficiles que doit subir un nouveau commissaire concerne ce que Goffman appelle « la gestion de son image ». Plus exactement, chacun d'entre eux se trouve face au défi consistant à apprendre une forme de représentation nouvelle au sein d'une institution multiculturelle qui se trouve en face d'une multiplicité d'espaces politiques. Or, lorsqu'on rappelle l'importance de la représentation pour la légitimation du pouvoir, ces difficultés participent pleinement à la faible institutionnalisation du rôle de commissaire. Comme le souligne l'anthropologue Marc Abélès, dans chaque société un acteur politique a la charge de « renvoyer à la collectivité qu'il incarne l'image de la cohérence et de la cohésion. Le pouvoir représente, cela signifie qu'un individu ou un groupe se pose comme le porte-parole de l'ensemble. Mais le pouvoir représente, aussi, en tant qu'il met en spectacle l'univers dont il est issu et dont il assure la permanence " ${ }^{41}$. Si la recherche en sciences sociales commence à s'interroger sur les rapports entre la légitimité de l'Union européenne et celle de la Commission et de ses pratiques, force est de constater que ce rôle est le plus souvent abordé sous l'angle d'un problème de communication. Si une telle problématique est intéressante et comporte un certain nombre d'avantages, il nous semble indispensable de la compléter par une interrogation sur le rapport qu'entretiennent les agents de la Commission, notamment les commissaires, avec les exigences de la représentation politique. En effet, nous 
verrons que, pour les commissaires, choisir entre faire des efforts pour s'imposer personnellement comme intervenant dans le débat public ou, inversement, s'en remettre aux services, est ce qui fait d'eux des hommes politiques ou non. Le défi communicationnel : intéresser un public qui reste à inventer Quel est le public des commissaires? La réponse à cette question générale est loin d'être simple. Comme le souligne une série de travaux en communication politique ${ }^{42}$, contrairement aux ministres nationaux, un commissaire n'a pas en permanence en face de lui des médias structurés qui peuvent lui servir de miroir et qui l'obligent à respecter des formes ritualisées de représentation. De même, le seul instrument de sondage européen, Eurobaromètre, n'est pas autorisé à poser des questions qui viendraient à comparer la perception publique des commissaires ${ }^{43}$. Face à cette incertitude quant à sa propre performance, chaque commissaire donne l'apparence de bricoler sa pratique communicationnelle comme il le peut. A partir de ce constat général, deux questions nous permettent de mieux comprendre quelques régularités au sein de ce bricolage : comment les commissaires cherchent-ils à intéresser les médias? Comment font-ils pour se positionner par rapport à d'autres acteurs du gouvernement européen qui sont, à priori, plus politiquement légitimes? Comme en témoignent les travaux en cours d'Olivier Baisnée $e^{44}$, pour un commissaire européen intéresser les médias n'a rien d'évident. Cette difficulté tient sans doute autant aux pratiques de ces acteurs et de leurs entourages qu'à la faible structuration de la presse, de la radio et de la télévision au niveau de l'Europe. Sous le premier angle, sauf exception, il ne fait pas de doute que les agents de la Commission tendent à produire et à diffuser un type d'information sur les politiques communautaires, actuelles ou futures, qui gomme le jeu politique qui les a engendrées. Soit sous forme de discours écrits, soit sous celle de communiqués de presse, le mode de communication dominant des commissaires s'explique en partie par leur positionnement, souvent délicat, au sein des négociations infra et extracommunautaires. Tiraillés entre leurs fonctions de négociateur et de représentant, bien des commissaires finissent par minimiser la seconde en faveur de la première. Pour en donner une illustration plus précise, en participant pleinement à la routinisation de la dissémination de l'information de la Commission, la nature " politiquement asexuée » ${ }^{45}$ des communiqués et des conférences de presse quotidiennes peut même renforcer la tendance des médias généralistes à négliger les activités des commissaires. Ce caractère diplomatique de la communication de la Commission incite Olivier Baisnée à analyser la presse accréditée à Bruxelles comme «le premier public » des commissaires et, plus largement, de la Commission dans son ensemble ${ }^{46}$. En effet, le Service des porte-parole a beau se vanter de nourrir tous les midis la plus grande salle de presse du monde (800 titres), force est toutefois de reconnaitre que ce type de communication attire un public dominé numériquement par les médias spécialisés et animés, du moins jusqu'à récemment, par quelques journalistes plus aptes à prôner la ligne de la Commission que de la mettre en débat et donc en scène. En effet, au-delà de ce premier public, les journalistes à Bruxelles ne sont pas toujours assurés de pouvoir " placer leurs papiers " en convaincant les chefs de rubrique et/ou les rédacteurs en chef à Paris ou à Londres de leur intérêt $t^{47}$. Plus exactement, en règle générale, de tels journalistes ont plus de chances de se faire publier dans la rubrique « économie » que dans les pages consacrées aux affaires que les médias qualifient de "politiques». En effet, en interviewant les membres des entourages des commissaires, nous avons été frappés par le sentiment de déception, voire même de trahison, qu'inspire le manque de reconnaissance accordée par les médias à leur commissaire. « En cinq ans de travail sur un dossier crucial, on n'a 
fait la une des infos de 20 heures qu'une fois.... » s'est lamenté, par exemple, un membre de cabinet d'Yves de Silguy au cours d'un entretien ${ }^{48}$. Certes, certains commissaires peuvent toujours essayer d'accéder directement aux pages des journaux en accordant des interviews plus ou moins exclusives. Toutefois, cette pratique connaît, elle aussi, des limites dans la mesure où la plupart des commissaires préfèrent faire passer des messages via les médias "fiables", tel que The Financial Times (cas de Leon Brittan et de Yves de Silguy), que de se risquer à dévoiler ce qu'ils font, et à quel titre, dans les pages de journaux plus populaires. Représentation et contraintes de rôle Ces contraintes qui pèsent sur les capacités à représenter des commissaires ne se résument pourtant pas à des conditions de communication que partagent finalement l'ensemble des acteurs européens. Le contexte institutionnel dans lequel ils déploient leur action constitue en effet une autre variable sur laquelle il convient de s'arrêter. Les commissaires ont à affronter un triple défi en la matière. Celui de la conciliation des règles de la collégialité, des rapports de la Commission avec le Conseil des ministres et des effets découlant de la nature multiculturelle de la Commission. Les exigences contradictoires qui en découlent tendent à brouiller le contenu et les frontières du rôle de représentant qu'ils sont susceptibles d'endosser. Au niveau du collège, les divergences constatées dans les approches de l'information sont caractéristiques des règles du jeu implicites gouvernant les rapports entre commissaires. Ces règles offrent une clé pour comprendre le fonctionnement particulièrement sectorialisé de la Commission dans sa globalité, et dans son approche de la communication en particulier. D'abord, on l'a vu, la réussite d'un commissaire dans la gestion de son portefeuille dépend fortement de sa capacité à nouer des alliances au sein du collège et donc à jouer le jeu de la collégialité - de l'Europe - plutôt que celui de la défense d'intérêts particuliers, nationaux notamment. Pour Ray Mac Sharry, par exemple, ces contraintes ont nui à son image dans son pays d'origine et l'ont placé en porte-à-faux vis-à-vis des intérêts des petits exploitants agricoles dont il s'estimait pourtant le porte-drapeau avant son entrée à la Commission ${ }^{49}$. Le jeu des contradictions qui pèsent, pour chaque commissaire, sur la définition de son image de représentant, est encore accentué par l'absence de toute incitation à l'harmonisation des stratégies en la matière. Ici aussi, la nécessité de ne pas s'attirer l'hostilité de ses partenaires tend à laisser chacun libre de ses choix en la matière: "La règle implicite pour un commissaire c'est : 'je fous la paix aux autres sur leur budget de communication afin de maximiser les chances d'accroître le mien' (...) au collège, il n'y a jamais de débat sur comment nous communiquons. Et d'ailleurs on ne se donne pas des moyens d'analyse non plus. On reste au niveau des conversations de café du commerce ${ }^{50}$. «Opinions », " intérêts ", " projets » sont autant de dimensions autour desquelles s'articule l'activité de représentation des commissaires, sans que le collège fournisse un quelconque vade mecum permettant d'en codifier les usages. Mieux, l'opportunité de la mise en scène de leur action est laissée à l'appréciation de chacun. Cette difficulté à se faire le porteparole de la Commission renforce un deuxième problème auquel doit faire face tout commissaire : la représentation que lui renvoient les acteurs politiques «légitimes", c'est-à-dire les ministres nationaux. Même si leur statut et leur rôle sont très différents, commissaires et ministres se trouvent souvent en concurrence pour attirer l'attention des journalistes. Or, vu la structuration essentiellement nationale des médias en Europe, ainsi que l'aura qui est le plus souvent attachée aux membres d'un gouvernement d'un pays membre, les commissaires tendent fortement à se trouver représentés comme des simples techniciens au service des « vrais » hommes politiques. 
Yves de Silguy et le traitement qui lui est réservé par les médias aux réunions mensuelles du Conseil des ministres de l'économie et des finances (ECOFIN) illustrent ce phénomène. En dépit du travail fait par de Silguy pour préparer et animer ces réunions, et, plus largement, assurer une permanence dans la mise en place d'une monnaie unique, au cours des conférences de presse qui ont suivi chaque Conseil, il s'est trouvé dans un rôle secondaire. Cette tendance renforce d'ailleurs l'importance des conférences de presse de la Commission, notamment celle du mercredi après la réunion hebdomadaire du collège. Un commissaire comme Yves de Silguy a beau s'en défendre («Après les réunions du Conseil, il fallait laisser parler la Présidence. Je savais qu'en cas de besoin je pourrais placer mon mot plus tard $»^{51}$ ), mais il n'en reste pas moins que les actions des ministres qui cherchent à technocratiser les commissaires, et plus généralement la Commission, constituent une partie intégrale d'une concurrence interinstitutionnelle peu équilibrée. Enfin, au-delà de ces contraintes infra et interinstitutionnelles, notre étude des défis de représentation des commissaires tend fortement à confirmer que ceux-ci sont moins liés à la non-élection des commissaires qu'à leur a-territorialité. Une analyse actuellement en cours de la fréquence, des lieux et du contenu des discours publics prononcés par les commissaires permettra au futur d'étayer cette hypothèse. Aujourd'hui nous pouvons néanmoins affirmer avec une certaine conviction qu'en développant ses pratiques de représentation, un commissaire est forcément confronté au dilemme suivant : représente-t-il l'Union européenne ? Si oui, quel contenu et quel sens lui accorder? Ou représente-t-il, malgré tout, un pays membre? Et, dès lors, comment développer une image européenne? Un commissaire court en permanence le risque de ne pas "sonner juste " en proposant à un public multiculturel des discours et des pratiques qui, faute d'un « fond de jeu » symbolique ${ }^{52}$, peinent à démontrer leur « sens social ${ }^{53}$.

2 Ce dernier point sert de rappel : notre analyse des commissaires aurait peu d'intérêt si nous ne l'avions pas inscrite dans une approche plus globale du gouvernement européen et de ses problèmes de légitimité. Ainsi, l'ouvrage qui clora notre recherche abordera la manière dont l'ambiguïté du rôle de commissaire révèle, de manière concrète, les contours et les effets d'un ordre institutionnel si faiblement ancré et impliqué dans une multiplicité d'espaces politiques. En ce qui concerne le présent texte, rappelons en conclusion que son contenu a été fortement orienté par un souci d'ordre théorique et méthodologique, consistant à concilier approche de sociologie de la politique et analyse de l'action publique. En insistant sur les choix préliminaires qui sont à faire pour réaliser une telle ambition dans le cas des commissaires, nous avons développé une démarche comportant trois entrées, fortement enchevêtrées, autour d'une seule question: quel est le rapport que les commissaires entretiennent à leur propre fonction? Tout d'abord, plutôt que de s'en tenir aux interrogations classiques d'une sociographie des élites (les sources de leur recrutement), notre analyse des trajectoires des commissaires et de leurs cabinets vise essentiellement à cerner la dynamique interne, voulue et réelle, des équipes que sont les commissaires. Ensuite, en informant l'équilibre développé entre capacités d'intervention sectorielle ( portefeuille ») et transversale (« hors portefeuille »), les compétences valorisées par chaque équipe constituent un élément d'explication de ce que font les commissaires. En orientant le rapport que chaque commissaire développe avec les Directions générales de la Commission, ainsi qu'avec ses interlocuteurs externes (gouvernements nationaux, groupes d'intérêts, parlementaires européens, etc.), la sociologie et la structuration de chaque équipe permettent d'éclairer le rôle du politique dans la fabrication des 
politiques publiques proposées au Conseil par le collège. Enfin, en travaillant en permanence la relation, souvent épineuse, entre intérêts nationaux et européens, les commissaires sont appelés à s'engager dans la transmission d'informations sur l'activité et les orientations de leur institution. Toutefois, en étudiant ce processus plus sous l'angle de la représentation politique que sous celui de la sociologie de la communication, nous avons interrogé les contraintes subies par les commissaires, contraintes qui sont autant de l'ordre inter-culturel qu'inter-institutionnel. Au total, en soulignant l'apport pour l'étude de l'intégration européenne de problématiques et d'outils de recherche développés au fil des décennies par la sociologie politique, cet article a esquissé un défi autant théorique qu'empirique. Tout comme les commissaires ne peuvent pas être abordés comme les simples décalques de ministres nationaux, analyser l'institutionnalisation et la légitimation de la Commission implique de mettre en cause un certain nombre de clivages et de questions qui continuent à structurer l'étude de l'intégration européenne.

\section{NOTES}

1. Ch. Lequesne «La Commission européenne entre autonomie et dépendance », Revue Française de Science Politique, 46/3, juin 1996.

2. En cherchant une population représentative à travers le croisement de diverses caractéristiques (petit/grand pays, droite/gauche, homme/femme, portefeuille fort/ portefeuille faible), nous avons retenu les individus suivants : Sir Leon Brittan, Edith Cresson, Yves de Silguy, Franz Fishler et Manuel Oreja Aguirre du collège 1995-99 et Sir Leon Brittan, Ray MacSharry, Christiane Scrivener pour celui de 1989-92. A ce jour, une soixantaine d'entretiens ont été effectués auprès d'anciens commissaires, d'agents de la Commission (porte-parole des commissaires, membres de cabinets, agents de DG), et de fonctionnaires du Parlement européen. Nous avons également dépouillé les discours publics écrits des commissaires étudiés. Cette recherche a été financée par le programme du CNRS «Identité européenne en questions ».

3. E. Page et L. Wouters, "Bureaucratic Politics and Political Leadership in Brussels », Public Administration 72, automne 1994 et E. Page People who run Europe, Oxford, Clarendon, 1997.

4. On s'inspire ici librement d'une formulation déjà exploitée par F. Sawicki sur un tout autre objet. Cf. F. Sawicki, « Laurent Fabius : du Giscard de gauche au socialisme moderne ", Pôle Sud 1, 1994, p. 37.

5. Ce qui ne va pas sans difficulté, le personnel ministériel étant relativement peu analysé par les politistes. Citons une des rares synthèses comparatistes sur ce point : J. Blondel et J.-L. Thiebault (dirs.), The Profession of Government Minister in Western Europe, Londres, Macmillan, 1991.

6. Ce type d'information est néanmoins accessible grâce à l'initiative de membres des services de documentation de la Commission qui assurent une mise à jour. Nous tenons tout particulièrement à remercier les personnes qui nous ont permis d'y avoir accès. 
7. O. Lewandowski, « Différenciation et mécanisme d'intégration de la classe dirigeante. L'image sociale de l'élite d'après le Who's who in France », Revue Française de Sociologie, 15/1, 1974.

8. M. Offerlé, « Professions et profession politique », in M. Offerlé (dir.), La profession politique XIXe-XXe siècles, Paris, Belin, 1999, p. 18.

9. E. Suleiman et H. Mendras (dirs.), Le recrutement des élites en Europe, Paris, La Découverte, 1995.

10. E. Page 1997, op. cit., p. 120.

11. A condition de bien vouloir passer outre les disparités que recouvrent ces catégories dans les pays concernés.

12. M. Abélès, La vie quotidienne au Parlement européen, Paris, Hachette, 1992, p. 69.

13. F. Sawicki, «Classer les hommes politiques. Les usages des indicateurs de position sociale pour la compréhension de la professionnalisation politique » in M. Offerlé (dir.), La profession politique, XIXe-XXe siècles, Paris, Belin, 1999.

14. Les $4 / 5$ des commissaires ont eu une expérience politique avant leur nomination, tandis que plus de la moitié $(56,2 \%)$ ont été ministres selon E. Page 1997, op. cit., p. 119. L'auteur ne tient pas compte des dates d'entrée à la Commission alors qu'il serait intéressant de ventiler ces chiffres sur l'ensemble de la période ouverte par la nomination de la première commission en 1958.

15. M. Donnelly et F. Ritchie, «The College of Commissionners and their Cabinets » in G. Edwards, D. Spence (dirs.), The European Commission, London, Longman, 1994.

16. Tendance que le nouveau Président de la Commission, Romano Prodi, a tenté d'atténuer en demandant aux commissaires de constituer des cabinets plus plurinationaux (Déclaration de R. Prodi, Libération, 29.9.99).

17. D'ailleurs, il importe de noter que si un pourcentage important des porte-parole sont fonctionnaires européens (environ 50\% en 1998), les autres tendent fortement à être recrutés directement par le commissaire dans le monde du journalisme.

18. Ch. Meyer, « Political Legitimacy and the Invisibility of Politics : Exploring the European Union's Communication Deficit », Journal of Common Market studies 37/4, 1999 et Ch. Meyer « Towards a European public sphere ? Transnational investigative journalism and the European Commission's resignation ", communication au colloque Transnational Communication, Berlin, 28-30 novembre 1999.

19. $P$. Mathiot et $F$. Sawicki, « Les membres des cabinets ministériels socialistes en France (1981-1993). Recrutement et reconversion », Revue Française de Science Politique 49/1, 1999 et « Les membres des cabinets ministériels socialistes en France (1981-1993). Passage en cabinet et trajectoires professionnelles », Revue Française de Science Politique 49/2, 2000.

20. Cette mise en corrélation des caractéristiques sociales et politiques des membres des cabinets ministériels et de leurs modes d'action et de participation aux processus de décision est un des axes autour desquels s'articule la thèse, déjà ancienne, qu'Ella Searls a consacré aux cabinets ministériels de la $V^{\circ}$ République. Cf. E. Searls, PoliticoAdministrative Relations in the Fifth French Republic : A Study of Ministerial Cabinets, unpublished Ph. D. thesis, London School of Economics, 1976.

21. Cf. Mathiot et Sawicki 2000, op. cit., p. 19.

22. Entretien 25/01/00.

23. Entretien $10 / 03 / 00$.

24. A. Smith, « L'intégration européenne, un processus foncièrement politique.

L'exemple de la Commission », communication à l'atelier La Commission européenne : 
une administration politique ?, congrès de l'Association française de science politique, Rennes, septembre 1999.

25. L. Cram, Policy-Making in the European Union : Conceptual Lenses and the Integration Process, New York : Routledge, 1997. M. Cini, The European Commission. Leadership, Organisation and Culture in the EU Administration, Manchester, Manchester University Press, 1996 et A. Smith, « La Commission européenne et les fonds structurels : vers un nouveau modèle d'action? », Revue française de science politique, vol 46/3, 1996.

26. G. Ross, Jacques Delors and European Integration, New York, Oxford University Press, 1995 ; K. Endo, The Presidency of the European Commission under Jacques Delors. The Politics of Shared Leadership, Oxford, Oxford University Press, 1999 et H. Drake, Perspectives on a European Leader : Jacques Delors, London, Routledge, 2000.

27. Entretien 10/3/00.

28. Entretien 7/12/99.

29. Cf. G. Ross, op. cit. Et L. Cram, op. cit.

30. Entretien $17 / 5 / 00$.

31. D. Gaxie, «Economie des partis et rétributions du militantisme », Revue française de science politique 1, janvier 1977.

32. E. Page 1997, op. cit.

33. E. Cresson, Innover ou subir, Paris, Flammarion, 1998.

34. F. Bailey, Les règles du jeu politique, Paris, PUF, 1973.

35. En s'appuyant sur les « problèmes de gestion » qui ont abouti à la démission de la Commission Santer, Romano Prodi a demandé aux nouveaux commissaires de s'installer avec leurs cabinets dans les bureaux de leur Direction générale. Cette évolution est loin d'être accueillie favorablement par plusieurs agents de la Commission que nous venons d'interviewer. Un membre de la Cellule de prospective, par exemple, craint que les commissaires ne deviennent des « super Directeurs généraux » (entretien 7/12/99).

36. Par ailleurs, ce défi peut être redoublé dans les cas où un commissaire partage un dossier avec le président de la Commission (le cas de Oreja Aguirre pour le portefeuille « questions institutionnelles » dans le cadre de la préparation du traité d'Amsterdam). 37. Un commissaire qui n'a qu'un portefeuille semble a priori bénéficier de plusieurs avantages. Ce type de dossier tend fortement à être la responsabilité d'un Conseil des ministres (ex. Franz Fishler et le Conseil « agricole » ou Yves de Silguy et le Conseil «ECOFIN » qui se réunissent tous les deux au moins dix fois par an). Ces réunions fournissent des points fixes dans l'agenda de la Commission qui permettent de mobiliser les services des Directions générales concernées et de provoquer les décisions au niveau du collège.

38. Entretien $17 / 5 / 00$.

39. Entretien $9 / 12 / 99$.

40. Entretien $9 / 12 / 99$.

41. M. Abélès, « La mise en représentation du politique » in M. Abélès et H-P. Jeudy, Anthropologie du politique, Paris, Armand Colin, 1997, p. 247.

42. Ph. Schlesinger, «Changing Spaces of Political Communication : The Case of the European Union ", Political Communication 16/3, 1999 ; D. Kevin \& Ph. Schlesinger, «Can the European Union Become a Sphere of Publics? » in E. Erikson \& J. Fossum, Democracy in the European Union : Integration through Deliberation?, London, Routledge, 2000. 
43. Basée sur l'avis des journalistes, une telle comparaison, apparemment suivie de près par de nombreux commissaires, est publiée régulièrement dans l'hebdomadaire The Economist.

44. O. Baisnée, «La Commission dans l'oeil des journalistes », communication à l'atelier La Commission européenne : une administration politique ?, congrès de l'Association française de science politique, Rennes, septembre 1999 et « Can Political Journalism Exist at the European Level ? ", communication à l'atelier « Political Journalism : New challenges, New practices », ECPR joint sessions, Copenhague, avril 14-19, 2000.

45. C. Schickel, Les journalistes accredités auprès de l'Union européenne, mémoire de DEA, IEP de Strasbourg, 1995, p. 46.

46. O. Baisnée 2000, op. cit.

47. C. Schickel, op. cit., 80 et s. Comme le souligne Olivier Baisnée, du moins dans la presse française et britannique, « controversies that are presented are those that can be translated into the traditional patterns of national politics » (O. Baisnée 2000, op. cit., p. 3). «In fact, the only conflicts that are given time and space are those involving Member States as is the case for any form of intergovernmental bargaining. While EU correspondants are very well aware of the intense political life in Brussels, this particular European dimension is hardly ever explored which results in a reinforcement of the public belief that Europe is excessively technocratic. There is a distinct difference between what these journalists know about any decision or fact and what they actually write about it » (ibid, p. 10).

48. Entretien 3/11/99. A certains égards, cette déception marque aussi un grand nombre d'analyses de l'intégration européenne en sciences sociales. Tout comme chez Habermas, on y retrouve souvent une arrière pensée évolutionniste qui attend, avec plus ou moins de patience, l'émergence d'un « espace public européen ». Cf. A. Smith, " "L'espace public européen" : une vue (trop) aérienne », Critique internationale 2. Cf. aussi l'article d'Adrian Favell dans ce numéro pour une critique similaire.

49. Entretien 09/03/00

50. Entretien 14/12/98.

51. Entretien 8/12/99.

52. C. Lager, L'Europe en quête de ses symboles, Bruxelles, Euroclio, 1995.

53. M. Augé, Pour une anthropologie des mondes contemporains, Paris, Aubier, 1994, p. 84.

\section{INDEX}

Thèmes : Commission européenne

Index géographique : Bruxelles, Union européenne

Mots-clés : bureaucraties/ théorie des organisations, construction européenne, institutions politiques et agences, sociologie 